\title{
Fluid resuscitation via the rectum ameliorates hemodynamic disorders through adjusting aquaporin expression in an experimental severe acute pancreatitis model
}

\author{
RONGLI XIE ${ }^{1,2 *}$, JINLONG WANG $^{3 *}$, YI YAO $^{2}$, MENGZHI QI $^{2}$, SHUNWEI HUANG $^{2}$, ZHIFENG ZHAO $^{1}$, \\ YING CHEN $^{2}$, ZHITAO YANG ${ }^{2}$, HUIQIU SHENG ${ }^{2}$, JIAN FEI ${ }^{1}$, ENQIANG MAO ${ }^{2}$ and ERZHEN CHEN ${ }^{2}$ \\ Departments of ${ }^{1}$ General Surgery and ${ }^{2}$ Emergency, Ruijin Hospital Affiliated to Shanghai Jiao Tong \\ University School of Medicine, Shanghai 200025; ${ }^{3}$ Emergency Center of The Affiliated \\ Hospital of Xuzhou Medical University, Xuzhou, Jiangsu 221004, P.R. China
}

Received March 12, 2018; Accepted October 30, 2018

DOI: $10.3892 /$ etm.2018.6934

\begin{abstract}
Acute pancreatitis is an acute abdominal disease, with $10-20 \%$ of the cases deteriorating rapidly, accompanied by persistent organ failure and further development into severe acute pancreatitis (SAP). The aim of the present study was to investigate the mechanism of fluid resuscitation via the rectum in the early stages of SAP and the role of aquaporins (AQPs). An SAP model was constructed by injection of $5 \%$ sterile sodium taurocholate into the biliopancreatic duct of Sprague Dawley rats, and the mean arterial pressure (MAP) was continuously monitored via femoral artery catheterization. At $30 \mathrm{~min}$ after the construction of the SAP model, the rats in the fluid resuscitation groups were resuscitated with normal saline at a rate of $4 \mathrm{ml} / \mathrm{kg} / \mathrm{h}$ through the venous or the rectal route. The AQP and $\mathrm{Na}^{+}-\mathrm{K}^{+}$-ATPase levels, and the correlation of the MAP and colon AQPs at the early stages of SAP were analyzed. The results demonstrated that the mRNA level of AQP-3 and AQP-4 in the distal colon decreased significantly in the group subjected to fluid resuscitation via the rectum, while no significant differences were identified in the $\mathrm{Na}^{+}-\mathrm{K}^{+}$-ATPase levels of the colon in that group. Furthermore, a negative correlation was identified between the expression of AQPs and the MAP $(\mathrm{P}<0.01)$. Thus, fluid resuscitation via the rectum appears to ameliorate hemodynamic disorders through
\end{abstract}

Correspondence to: Dr Ying Chen, Department of Emergency, Ruijin Hospital Affiliated to Shanghai Jiao Tong University School of Medicine, 197 Ruijin 2nd Road, Shanghai 200025, P.R. China

E-mail: bichatlion@163.com

Dr Jian Fei, Department of General Surgery, Ruijin Hospital Affiliated to Shanghai Jiao Tong University School of Medicine, 197 Ruijin 2nd Road, Shanghai 200025, P.R. China

E-mail: feijian@hotmail.com

*Contributed equally

Key words: aquaporins, severe resuscitation, hemodynamic disorders adjusting the expression of AQP-3 and AQP-4 in the distal colon in an experimental SAP model.

\section{Introduction}

Acute pancreatitis (AP) is an acute abdominal disease caused by pancreatic enzyme activation through a variety of triggers, followed by a pancreatic local inflammatory response as the major clinical feature. In an estimate of $10-20 \%$ of patients with AP, the condition deteriorates rapidly and develops into persistent organ failure, further evolving into severe acute pancreatitis (SAP) $(1,2)$. In the early phases of the disease, the gastrointestinal tract is one of the major targets, with an overwhelming release of inflammatory cytokines and exudation of fluids, leading to a sharp decline of the effective circulatory blood volume, thereby affecting the permeability of the colonic mucosal epithelium and water metabolism. Clinical studies have reported that the mortality rate of SAP within 14 days was as high as $50 \%$ and that effective early intervention had a significant impact on the prognosis of SAP $(3,4)$. Chen et al $(5)$ demonstrated that fluid resuscitation via the rectum improved organ function and decreased the serum levels of inflammatory factors.

Aquaporins (AQPs) are integral membrane proteins that belong to the major intrinsic protein family. AQPs constitute channels in the cell membrane, which control the transportation of water and restrict the movement of ions or other micromolecules in and out of the cells $(6,7)$. AQPs, including AQP-1, -3, -7, -8 and -11, are abundantly expressed in the human colon tissue, and have a vital role in digestive disturbances, including diarrhea and constipation (8-11). A recent study (12) proved that the expression of AQPs in colon tissues increased significantly in SAP, but in a diverse manner. The aim of the present study was to investigate the potential beneficial effect of fluid resuscitation via the rectum in the early stages of SAP and the underlying mechanisms, including the role of AQPs.

\section{Materials and methods}

Chemicals and reagents. Sodium taurocholate (purity, $\geq 97 \%$ ) was purchased from Sigma-Aldrich (Merck KGaA, Darmstadt, 
Germany). PrimeScript RT Master Mix and SYBR Premix Ex Taq were purchased from Takara Bio Inc. (Otsu, Japan). Rabbit anti-rat $\mathrm{Na}^{+}-\mathrm{K}^{+}$-ATPase (cat. no. SC-28800) was purchased from Santa Cruz Biotechnology Inc. (Dallas, TX, USA).

Animals. Male Sprague Dawley (SD) rats (age, 8-10 weeks; weight, $300 \pm 10 \mathrm{~g}$ ) were purchased from the Shanghai Laboratory Animal Center (Chinese Academy of Science, Shanghai, China). The rats were provided rodent chow and tap water ad libitum for at least 1 week and were allowed to acclimatize to the laboratory environment. The rats were anesthetized via an intraperitoneal injection of 3\% pentobarbital sodium $(50 \mathrm{mg} / \mathrm{kg}$ body weight), and an additional injection of $1 / 5$ of the initial dose was given every hour throughout the entire experiment. Clinical signs, including the mean arterial pressure (MAP), were continuously monitored with a multi-channel physiological recorder (Powerlab 15T; ADInstruments, Bella Vista, NSW, Australia). The rats were sacrificed by $\mathrm{CO}_{2}$ inhalation in accordance with the institutional criteria for euthanasia.

SAP model establishment and fluid resuscitation. The SAP model was established as previously described (5). The rats were fasted overnight with free access to water prior to the experiment, and were randomly divided into four groups as follows: The SHAM, no fluid resuscitation (NFR), intravenous fluid resuscitation (IVFR) and fluid resuscitation via the rectum (FRVR) groups. SAP was induced by injection of $5 \%$ sterile sodium taurocholate (final dose, $0.1 \mathrm{ml} / 100 \mathrm{~g}$ ) into the biliopancreatic duct of the SD rats, and the MAP was continuously monitored via femoral artery catheterization throughout the experiment. At $30 \mathrm{~min}$ after the induction of SAP, the rats in the IVFR and FRVR groups were resuscitated with normal saline at a rate of $4 \mathrm{ml} / \mathrm{kg} / \mathrm{h}$ through the venous or the rectal route (5), while the rats in the NFR group were not administered any fluid therapy. In the rats of the SHAM group, catheterization and laparotomy were performed, and the duodenum and pancreas were flipped instead of inducing SAP. At $12 \mathrm{~h}$ after the beginning of fluid resuscitation, the rats were sacrificed by $\mathrm{CO}_{2}$ inhalation, except for model rats that were sacrificed at 4, 8, 12 and $24 \mathrm{~h}$ for the determination of $\mathrm{Na}^{+}-\mathrm{K}^{+}$-ATPase.

Detection of AQP-3, AQP-4 and AQP-8 mRNA levels by reverse transcription-quantitative polymerase chain reaction $(R T-q P C R)$ analysis. Total RNA was extracted from colon tissues using TRIzol reagent (Thermo Fisher Scientific, Inc.). Complementary DNA was synthesized by RT according to the protocol of PrimeScript RT Master Mix and then subjected to qPCR using $\beta$-actin as an internal control. The primer sequences were as follows (5'-3'): AQP-3 forward, CCCCTT GTGATGCCTCTC and reverse, CCCTAGCTGGCAGAG TTC; AQP-4 forward, CAGAACCAAGGCGTAGACCG and reverse, TCCCTGGAAATGACTGAGAAA; AQP-8 forward, GCCGATGTGTAGTATGGACCTA and reverse, ACCCAA TGAAGATGAAGAGAGC; $\beta$-actin forward, GCGCTCGTC GTCGACAACGG and reverse, GTGTGGTGCCAAATCTTC TCC. The PCR was performed according to the manufacturer's protocol using an Applied Biosystems 7500 Real-Time PCR System (Thermo Fisher Scientific, Inc.). The thermocycling protocol was as follows: Denaturation for $30 \mathrm{sec}$ at $95^{\circ} \mathrm{C}$ and 40 cycles of $5 \mathrm{sec}$ at $95^{\circ} \mathrm{C}$ and $34 \mathrm{sec}$ at $60^{\circ} \mathrm{C}$. The relative changes in gene expression were calculated as $2^{-\Delta \Delta \mathrm{Cq}}(13)$.

ELISA detection of $\mathrm{Na}^{+}-\mathrm{K}^{+}$-ATPase. The concentration of $\mathrm{Na}^{+}-\mathrm{K}^{+}$-ATPase was determined using ELISA kits according to the manufacturer's protocol (cat. no. SC-28800; Santa Cruz Biotechnology Inc.).

Sample staining. Histopathological examination was performed using the hematoxylin and eosin (H\&E) staining method. Fresh tissue specimens were immersed in $4 \%$ paraformaldehyde for $12 \mathrm{~h}$ at room temperature. Samples were dehydrated in room temperature through a serial alcohol gradient $(50 \%, 3 \times 1 \mathrm{~h} ; 70 \%, 1 \mathrm{~h} ; 80 \%, 1 \mathrm{~h} ; 90 \%, 1 \mathrm{~h} ; 100 \%$, $2 \times 1 \mathrm{~h})$, followed by immersing in xylene $(2 \times 20 \mathrm{~min})$ at room temperature and embedded in paraffin wax blocks $\left(52-56^{\circ} \mathrm{C}\right.$; $3 \times 2 \mathrm{~h})$. Paraffin-embedded tissue sections $(4 \mu \mathrm{m})$ were prepared and dewaxed in xylene (3x $10 \mathrm{~min}$ at room temperature), rehydrated through decreasing concentrations of ethanol (100\%, 2x10 min; 90\%, $5 \mathrm{~min}$; 80\%, $5 \mathrm{~min}$; 70\%, $5 \mathrm{~min}$ ), and washed with PBS. Samples were stained using the H\&E staining kit according to the manufacturer's protocol (Sangon Biotech Co., Ltd., Shanghai, China) at room temperature for $10 \mathrm{~min}$ and $30 \mathrm{sec}$, respectively. Samples were observed under a light microscope (magnification, x100).

Statistical analysis. Values are expressed as the mean \pm standard error of the mean and compared using Student's t-tests or one-way analysis of variance with Student-Newman-Keuls post hoc test. Pearson correlation analysis was performed to assess the correlation between the MAP and the mRNA levels of AQPs. P<0.05 was considered to indicate a statistically significant difference. Analysis was performed using SPSS version 19 (IBM Corp., Armonk, NY, USA) and Prism (version 5.0; GraphPad Software, Inc., La Jolla, CA, USA).

\section{Results}

Pathological changes of the colon. After the establishment of SAP, the integrity of the proximal colonic mucosa was continuously damaged and inflammatory cell infiltration was observed in the submucosa. Compared with the SHAM group, the structure of the epithelial cells was disturbed and the colonic villi were destroyed in the NFR group (Fig. 1A and B). Following IVFR, the condition of the lesions improved significantly (Fig. 1C), with only mild edema observed in the proximal colon, whereas the inflammatory cell infiltration had subsided. The pathological changes in the distal colon were almost identical to those in the proximal colon (Fig. 1E, F). Similarly, between distal and proximal colon, fluid resuscitation via the rectum also effectively reduced the inflammatory response (Fig. 1D and $\mathrm{H}$ ).

Changes in the mRNA levels of colonic AQPs with fluid resuscitation in SAP. The expression of AQP-3, AQP-4 and AQP-8 mRNA in the proximal and distal colon was significantly higher compared with that in the SHAM group (Fig. 2). In the proximal colon, the mRNA levels of AQP-3 and AQP-8 in the 


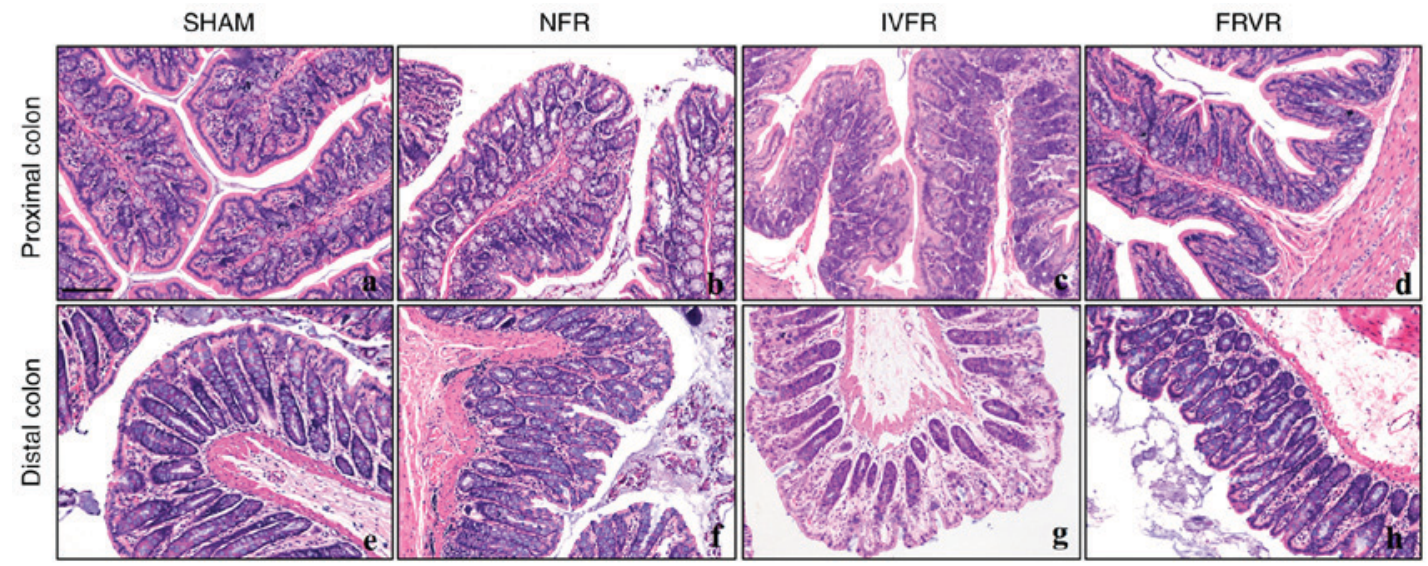

Figure 1. Pathological changes in the colon. (a) SHAM, (b) NFR, (c) IVFR and (d) FRVR groups in the proximal colon. The group of (e) SHAM, (f) NFR, (g) IVFR and (h) FRVR in the distal colon. Histopathological examination was performed using the hematoxylin and eosin staining method (magnification, x100). NFR, no fluid resuscitation; IVFR, intravenous fluid resuscitation; FRVR, fluid resuscitation via the rectum.

IVFR and FRVR groups were significantly lower compared with those in the NFR group, while they were still higher compared with those in the SHAM group. Following fluid resuscitation, a different response was observed regarding the changes in the levels of AQP-4. The expression of AQP-4 mRNA in the IVFR group was higher compared with that in the NFR group, but was decreased in the FRVR group.

The results obtained in the distal colon were different from those in the proximal colon. Following fluid resuscitation, although the expression of AQP-8 mRNA was higher compared with that in the SHAM group, there was no significant difference between the NFR group and the resuscitation groups. Furthermore, FRVR effectively reduced the levels of AQP-3 and AQP-4, while IVFR did not. Of note, the expression of AQP-4 mRNA was lower compared with that in the SHAM group.

Changes in colon $\mathrm{Na}^{+}-\mathrm{K}^{+}$-ATPase at the early stages of $S A P . \mathrm{Na}^{+}-\mathrm{K}^{+}$-ATPase is an important ion channel on the cell membrane. It is used to transport sodium and potassium ions through an inverse concentration gradient to help the transmembrane diffusion of water. Thus, the expression of $\mathrm{Na}^{+}-\mathrm{K}^{+}$-ATPase was investigated in the colon tissues of SAP rats (Fig. 3). In the SHAM group, the expression levels of $\mathrm{Na}^{+}-\mathrm{K}^{+}$-ATPase in the proximal and distal colon were similar. Furthermore, compared with the SHAM group, the expression of $\mathrm{Na}^{+}-\mathrm{K}^{+}$-ATPase in the SAP model maintained for $12 \mathrm{~h}$ (S12h group) was significantly higher $(\mathrm{P}<0.05)$. In the $\mathrm{S} 24 \mathrm{~h}$ group, the results were different between the proximal and distal colon: The proximal colon exhibited a significant increase of $\mathrm{Na}^{+}-\mathrm{K}^{+}$-ATPase compared with that in the $\mathrm{S} 12 \mathrm{~h}$ group, but a significant decrease in the distal colon. Following fluid resuscitation, the $\mathrm{Na}^{+}-\mathrm{K}^{+}$-ATPase expression in the proximal colon of the IVFR group was significantly reduced compared with that in the SHAM and NFR groups $(\mathrm{P}<0.05$; Fig. $3 \mathrm{C}$ ) while there is no difference between the FRVR and NFR groups. However, no significant differences were observed in the $\mathrm{Na}^{+}-\mathrm{K}^{+}$-ATPase levels in the distal colon among the four groups ( $\mathrm{P}>0.05$; Fig. 3D).

Correlation of MAP with colon AQP expression at the early stages (12 $h$ following onset) of SAP (3/group). A negative correlation was observed between AQP expression and the MAP (Fig. 4). In addition, AQP expression in the proximal colon was more significantly correlated with the MAP compared with that in the distal colon of SAP rats. In proximal colon tissues, the correlation analysis between the mRNA levels of AQP-3, -4 and -8 and the MAP provided $r$ values of $-0.9124,-0.9335$ and -0.9345 , respectively (Fig. 4A-C). In distal colon tissues, the $\mathrm{r}$ values were $-0.8228,-0.8847$ and -0.8831 , respectively (Fig. 4D-F).

\section{Discussion}

SAP is an inflammation of the pancreas that is associated with a significant amount of morbidity and mortality. According to clinical experience, there are two mortality rate peaks over the course of SAP, with most deaths occurring in the early stages of the acute response, which is considered to be the primary factor for the rapidly aggravated early systemic disease leading to death. Hence, effective correction of systemic circulation and microcirculation disorders shortens the time of tissue hypoxia and control of sustained systemic inflammatory response syndrome is key to the treatment of the acute response $(14,15)$. The most effective treatment is fluid resuscitation, which is recognized as the primary measure in the current guidelines $(2,16)$. Chen et al $(5)$, demonstrated that FRVR is a potential supplementary method for fluid management at the early stages of SAP. Following treatment with FRVR, the MAP and organ function improved, suggesting that the colon may readily absorb a large volume of water under conditions of shock. Hence, the aim of the present study was to investigate the mechanisms underlying water absorption through the colon during SAP.

AQPs are widely distributed in the cell membranes of human tissues and visceral organs, and participate in almost all pathophysiological processes of a variety of diseases $(6,17)$. A number of studies revealed that AQP-3, -4 and -8 are implicated in water uptake and transport in the colon. AQPs may participate in the adjustment of intestinal mucosal permeability and the rate of water absorption. Abnormalities of AQPs are known to be associated with Crohn's disease, ulcerative colitis, slow transit constipation and cholera (18-24). A previous 

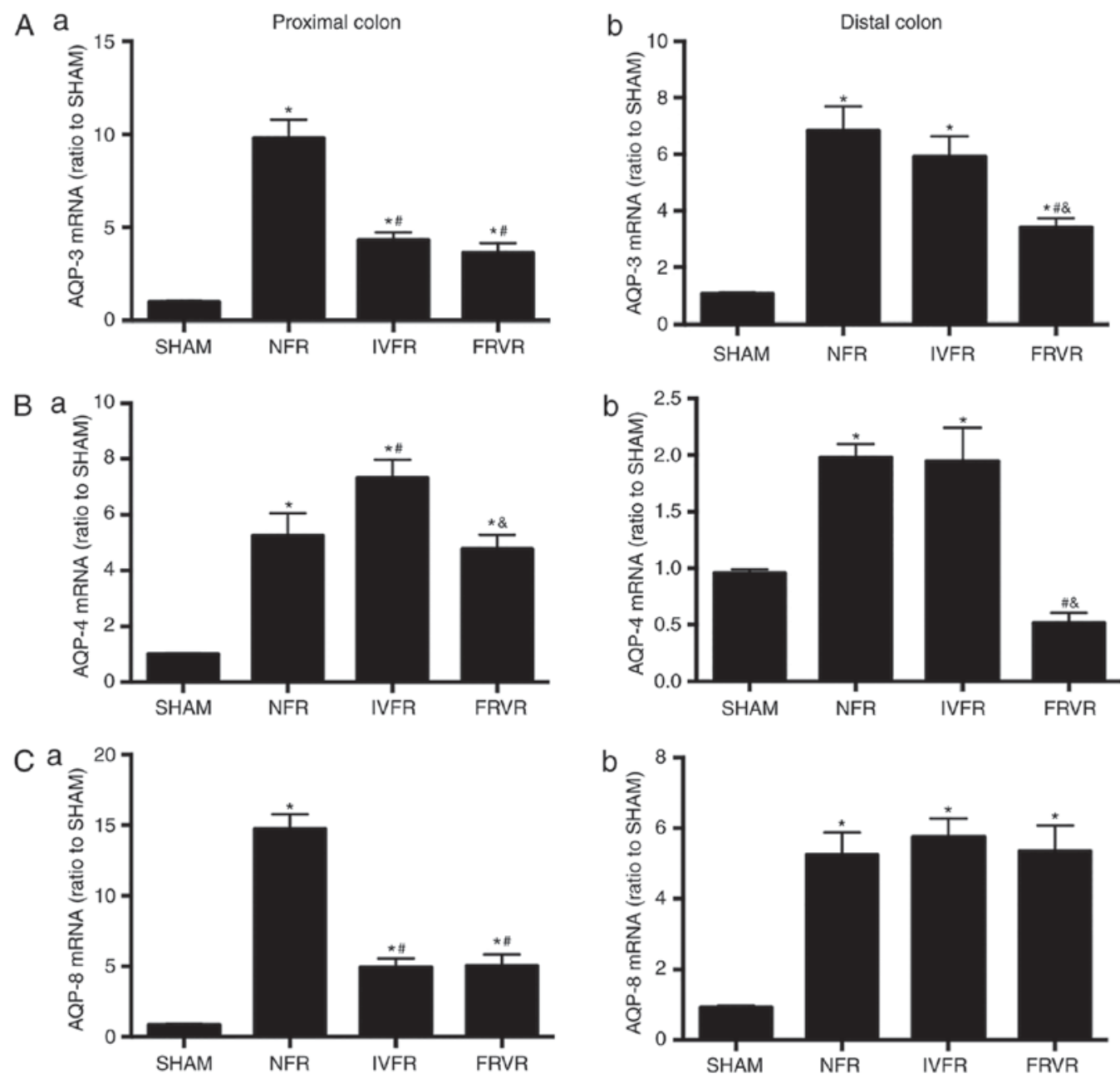

Figure 2. Changes in the mRNA levels of colonic AQPs with fluid resuscitation in severe acute pancreatitis. (A) AQP-3, (B) AQP-4 and (C) AQP-8 in (a) the proximal and (b) distal colon. ${ }^{*} \mathrm{P}<0.05$ vs. SHAM group, ${ }^{*} \mathrm{P}<0.05$ vs. NFR group, ${ }^{\circledR} \mathrm{P}<0.05$ vs. IVFR group. NFR, no fluid resuscitation; IVFR, intravenous fluid resuscitation; FRVR, fluid resuscitation via the rectum; AQP, aquaporin.
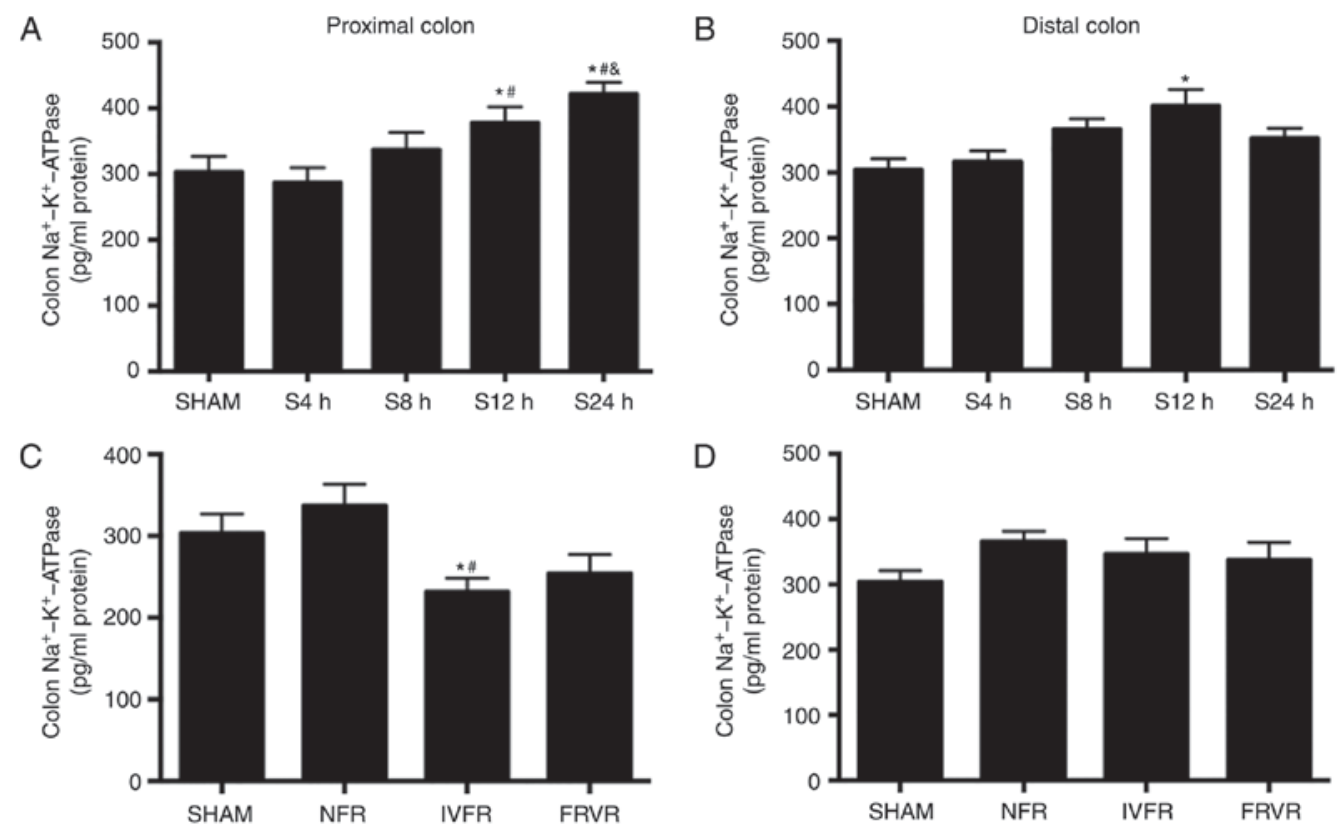

Figure 3. Changes in colon $\mathrm{Na}^{+}-\mathrm{K}^{+}$-ATPase at the early stages of SAP. (A and B) $\mathrm{Na}^{+}-\mathrm{K}^{+}$-ATPase levels in the (A) proximal and (B) distal colon of the SAP model group over $24 \mathrm{~h}$. ${ }^{*} \mathrm{P}<0.05$ vs. SHAM group; ${ }^{\sharp} \mathrm{P}<0.05$ vs. S4h group; ${ }^{\circledR} \mathrm{P}<0.05$ vs. S8h group. (C and D) Na ${ }^{+}-\mathrm{K}^{+}-\mathrm{ATPase}$ levels in the $(\mathrm{C})$ proximal and (D) distal colon of the different experimental groups. ${ }^{*} \mathrm{P}<0.05$ vs. SHAM group; ${ }^{\#} \mathrm{P}<0.05$ vs. NFR group. SAP, severe acute pancreatitis; S4h, SAP model maintained for $4 \mathrm{~h}$; NFR, no fluid resuscitation; IVFR, intravenous fluid resuscitation; FRVR, fluid resuscitation via the rectum. 
A

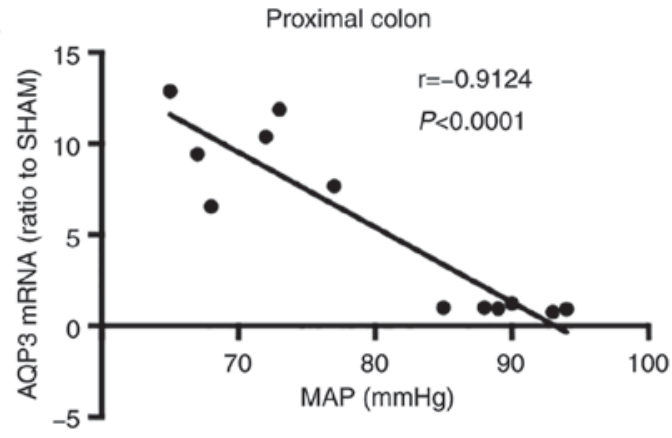

C

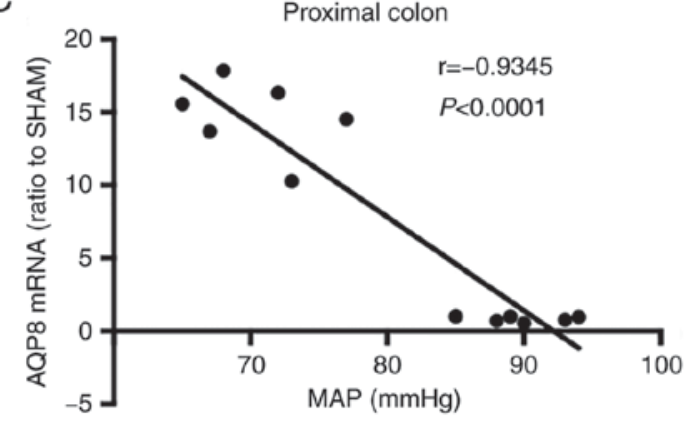

E

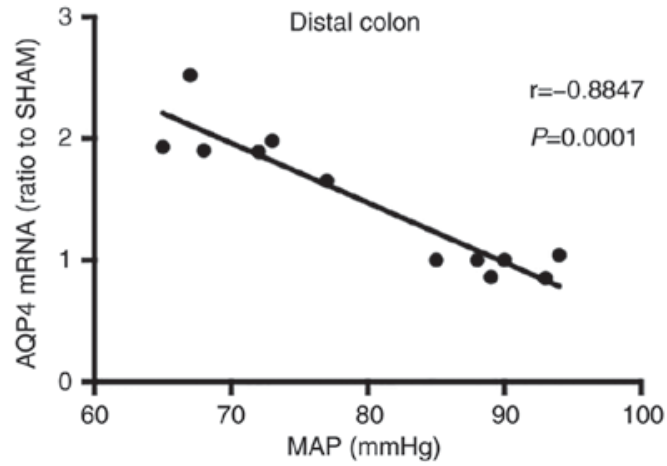

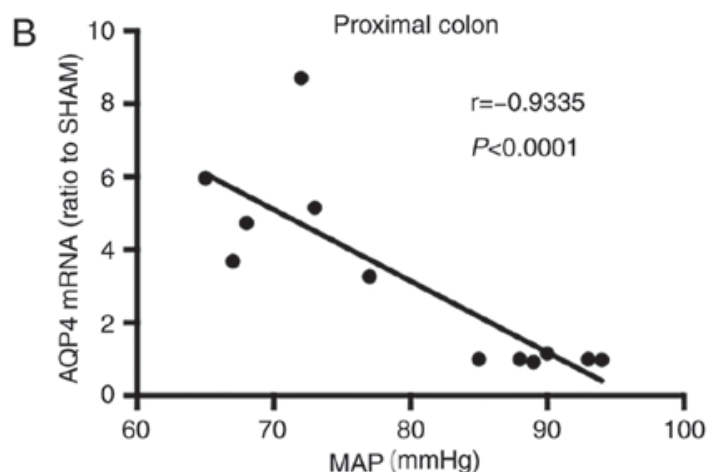
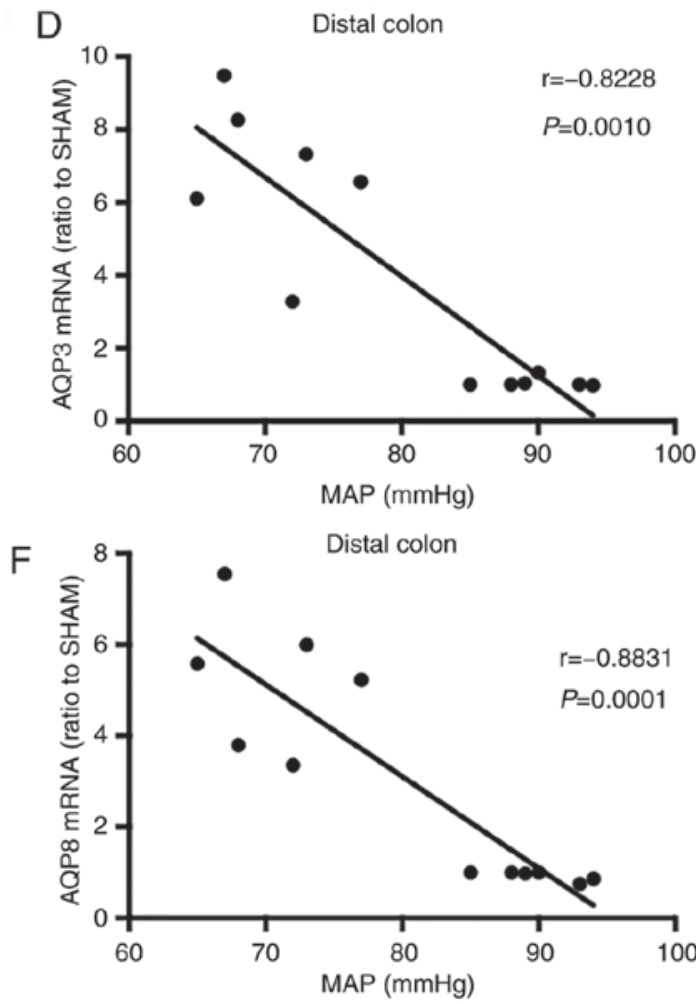

Figure 4. Correlation of MAP with colonic AQP expression at an early stage (12 h following onset) of severe acute pancreatitis. MAP was continuously monitored via femoral artery catheterization. In the proximal colon tissues, the $\mathrm{r}$ of (A) AQP3, (B) AQP4 and (C) AQP8 was $-0.9124,-0.9335$ and -0.9345 , respectively $(\mathrm{P}<0.0001)$. In the distal colon tissues, the $\mathrm{r}$ of $(\mathrm{D}) \mathrm{AQP}$, (E) AQP4 and (F) AQP8 was $-0.8228(\mathrm{P}=0.0010),-0.8847(\mathrm{P}=0.0001)$ and -0.8831 $(\mathrm{P}=0.0001)$, respectively. MAP, mean arterial pressure; $\mathrm{AQP}$, aquaporin.

study (12) and the results of the present study demonstrated that the expression of AQPs in colon tissues during SAP increased significantly, but in a diverse manner, and a negative correlation between AQP expression and the MAP was determined. Thus, it may be hypothesized that a negative feedback mechanism exists, which improves the intestinal water intake ability in order to restore the blood volume. This may be the mechanism through which FRVR exerts a therapeutic effect in SAP.

Compared with the jejunum and ileum, AQP-3 is abundantly expressed in the colon. Silberstein et al (25), initially discovered that AQP-3 was specifically expressed in epithelial microvilli of the colon, indicating that it may be involved in water absorption. In addition, suppression of AQP-3 function may lead to diarrhea by increasing the fecal water content. Furthermore, the purgative action of magnesium sulfate changes the osmotic pressure and downregulates AQP-3 expression in mucosal epithelial cells of the colon, resulting from activation of adenylate cyclase by increasing the intracellular magnesium ion concentration $(20,26)$. In the present study, normal rat colon tissues expressed low levels of AQP-3. With fluid resuscitation therapy, the level of colonic AQP-3 decreased in the proximal colon and distal colon.

A previous study indicated that $\mathrm{AQP}-4$ is mainly expressed in the ascending colon in humans (24). In rats, AQP-4 was reported to be predominantly expressed in the epithelial cells of the proximal colon $(21,27)$. Wang et al (22), studied AQP-4 gene knockout mice and identified that the expression of AQP-4 was positively correlated with the water permeability of the colon. In addition, high expression of AQP-4 accelerated water absorption through the colon, which may be a key event in slow transit constipation. AQP-4 was identified to be downregulated in a mouse model of allergic diarrhea, which was explained by a dysfunction of colonic absorption (21). In the present study, following treatment with invasive fluid resuscitation, the mRNA expression of AQP-4 was elevated; 
however, the expression was significantly decreased in the FRVR group, particularly in the distal colon.

AQP-8 is expressed in the colon and pancreas in humans. In rats, this protein was identified to be abundantly expressed in body parts including the duodenum, jejunum, rectum and liver (28-31). In the present study, the AQP-8 mRNA expression was altered not only in the IVFR group, but also in the FRVR group: Similar to AQP-3 and AQP-4, the expression of AQP-8 was increased in the NFR group. In the proximal colon, the expression decreased significantly in IVFR group and FRVR group, as expected. However, there was no significant change in distal colon following the fluid resuscitation.

In the early stages of SAP, the expression of AQPs was negatively correlated with the MAP, suggesting that, due to the significant loss of body fluids, hemodynamic abnormalities may increase the expression of colon AQPs. The upregulation of these proteins may constitute a negative feedback mechanism, enhancing the ability of the colon to absorb water in order to restore the effective circulatory volume. In the present study, the expression of AQP-3 and AQP-4 in the distal colon was only downregulated following rectal fluid resuscitation, whereas intravenous rehydration exerted a less prominent or no effect, suggesting that rectal administration of fluids to improve the colon hypoperfusion and restore the blood volume reduces the expression of AQPs in the distal colon. This result indicates that rectal fluid resuscitation may achieve active regulation of water absorption, while during invasive intravenous fluid resuscitation, the body only passively accepts the water. No significant change in distal colon AQP-8 was detected after fluid resuscitation, suggesting that AQP-8 exerted no effect on the regulation of distal colonic water absorption.

Various biological mechanisms participate in water absorption and transport. The present study also examined the association between $\mathrm{Na}^{+}-\mathrm{K}^{+}$-ATPase and SAP (pathological and recovery process). $\mathrm{Na}^{+}-\mathrm{K}^{+}$-ATPase is a vital ion channel, which facilitates the movement of water molecules across the cell membrane. $\mathrm{Na}^{+}-\mathrm{K}^{+}$-ATPase uses ATP for the intracellular and extracellular transport of sodium and potassium ions against a concentration gradient. The expression of $\mathrm{Na}^{+}-\mathrm{K}^{+}$-ATPase was analyzed in the present study. In the proximal and distal colon of the SHAM group, $\mathrm{Na}^{+}-\mathrm{K}^{+}$-ATPase was expressed at similar levels. During the pathological progression of SAP, the $\mathrm{Na}^{+}-\mathrm{K}^{+}$-ATPase increased with time, but fluid resuscitation had little or no effect on its expression, particularly in the distal colon except, for the IVFR group and the proximal colon.

SAP is a systemic inflammatory disease originating in the pancreas but involving multiple organs, and features a high mortality rate and multiple complications. Conventional treatment includes intravenous fluid therapy to improve the effective circulatory blood volume. However, a recent study (5) by our group indicated that fluid resuscitation via the rectum may decrease the occurrence of complications and, therefore, significantly improve the prognosis of affected patients. In the present study, it was demonstrated that fluid resuscitation via the rectum effectively reduced the inflammatory response in a rat model of SAP. Furthermore, the underlying mechanism was identified to be associated with AQPs rather than with $\mathrm{Na}^{+}-\mathrm{K}^{+}$-ATPase. This provides convincing evidence for the efficacy of fluid resuscitation via the rectum in the treatment of SAP and furthermore, AQPs may be a potential target in SAP research.

\section{Acknowledgements}

Not applicable.

\section{Funding}

The present study was supported by the National Natural Science Foundation of China (grant nos. 81670581, 81671901 and 81600501).

\section{Availability of data and materials}

The datasets used and analyzed during the current study are available from the corresponding author on reasonable request.

\section{Authors' contributions}

RX, JW, YC and JF designed the research. JW, YC, YY, MQ, $\mathrm{SH}$ and $\mathrm{ZZ}$ performed the research. RX, YC, ZY, HS, EM and EC analyzed the data. RX and YC prepared the manuscript. All of the authors listed have approved the final version of the manuscript submitted for publication.

\section{Ethical approval and consent to participate}

The Institutional Animal Care and Use Committee (IACUC) of Ruijin Hospital affiliated to Shanghai Jiao Tong University School of Medicine (Shanghai, China) approved the study protocol and the animal surgical procedures. All experimental procedures were performed according to the Guide for the Care and Use of Laboratory Animals developed by the Ruijin Hospital affiliated to Shanghai Jiao Tong University School of Medicine (Shanghai, China).

\section{Patient consent for publication}

Not applicable.

\section{Competing interests}

The authors declare that they have no competing interests.

\section{References}

1. Tenner S, Baillie J, DeWitt J and Vege SS; American College of Gastroenterology: American college of gastroenterology guideline: Management of acute pancreatitis. Am J Gastroenterol 108: $1400-1415,2013$

2. Banks PA, Bollen TL, Dervenis C, Gooszen HG, Johnson CD, Sarr MG, Tsiotos GG and Vege SS; Acute Pancreatitis Classification Working Group: Classification of acute pancreatitis-2012: Revision of the Atlanta classification and definitions by international consensus. Gut 62: 102-111, 2013.

3. Carnovale A, Rabitti PG, Manes G, Esposito P, Pacelli L and Uomo G: Mortality in acute pancreatitis: Is it an early or a late event? JOP 6: 438-444, 2005.

4. Jacob AO, Stewart P and Jacob O: Early surgical intervention in severe acute pancreatitis: Central Australian experience. ANZ J Surg 86: 805-810, 2016.

5. Chen Y, Ma L, Song X, Fei J, Chen E and Mao E: Beneficial effects of fluid resuscitation via the rectum on hemodynamic disorders and multiple organ injuries in an experimental severe acute pancreatitis model. Pancreatology 15: 626-634, 2015.

6. Agre P: The aquaporin water channels. Proc Am Thorac Soc 3: $5-13,2006$. 
7. King LS and Agre P: Pathophysiology of the aquaporin water channels. Annu Rev Physiol 58: 619-648, 1996.

8. Koyama N, Ishibashi K, Kuwahara M, Inase N, Ichioka M, Sasaki S and Marumo F: Cloning and functional expression of human aquaporin8 cDNA and analysis of its gene. Genomics 54: $169-172,1998$

9. Hasegawa H, Lian SC, Finkbeiner WE and Verkman AS Extrarenal tissue distribution of CHIP28 water channels by in situ hybridization and antibody staining. Am J Physiol 266: C893-C903, 1994.

10. Zahn A, Moehle C, Langmann T, Ehehalt R, Autschbach F, Stremmel W and Schmitz G: Aquaporin-8 expression is reduced in ileum and induced in colon of patients with ulcerative colitis. World J Gastroenterol 13: 1687-1695, 2007.

11. Tsujikawa T, Itoh A, Fukunaga T, Satoh J, Yasuoka T and Fujiyama Y: Alteration of aquaporin mRNA expression after small bowel resection in the rat residual ileum and colon J Gastroenterol Hepatol 18: 803-808, 2003.

12. Chen Y, Xie R and Wang J: Expression and role of aquaporin in the colon of acute necrotizing pancreatitis rats. Chin J Pancreatol 17: 162-167, 2017 (In Chinese).

13. Livak KJ and Schmittgen TD: Analysis of relative gene expression data using real-time quantitative PCR and the 2(-Delta Delta C(T)) method. Methods 25: 402-408, 2001

14. Guo Q, Li A, Xia Q, Liu X, Tian B, Mai G, Huang Z, Chen G Tang W, Jin X, et al: The role of organ failure and infection in necrotizing pancreatitis: A prospective study. Ann Surg 259: 1201-1207, 2014

15. Trikudanathan G, Navaneethan U and Vege SS: Current controversies in fluid resuscitation in acute pancreatitis: A systematic review. Pancreas 41: 827-834, 2012.

16. Zhou ZG, Chen YD, Sun W and Chen Z: Pancreatic microcirculatory impairment in experimental acute pancreatitis in rats. World J Gastroenterol 8: 933-936, 2002

17. Agre P and Kozono D: Aquaporin water channels: Molecular mechanisms for human diseases. FEBS Lett 555: 72-78, 2003.

18. Zheng YF, Liu CF, Lai WF, Xiang Q, Li ZF, Wang H and Lin N: The laxative effect of emodin is attributable to increased aquaporin 3 expression in the colon of mice and HT-29 cells. Fitoterapia 96: 25-32, 2014.

19. Kon R, Ikarashi N, Nagoya C, Takayama T, Kusunoki Y, Ishii M, Ueda H, Ochiai W, Machida Y, Sugita K and Sugiyama K: Rheinanthrone, a metabolite of sennoside A, triggers macrophage activation to decrease aquaporin-3 expression in the colon, causing the laxative effect of rhubarb extract. J Ethnopharmacol 152 190-200, 2014
20. Ikarashi $\mathrm{N}$ : The elucidation of the function and the expression control mechanism of aquaporin-3 in the colon. Yakugaku Zasshi 133: 955-961, 2013 (In Japanese).

21. Yamamoto T, Kuramoto $\mathrm{H}$ and Kadowaki M: Downregulation in aquaporin 4 and aquaporin 8 expression of the colon associated with the induction of allergic diarrhea in a mouse model of food allergy. Life Sci 81: 115-120, 2007.

22. Wang KS, Ma T, Filiz F, Verkman AS and Bastidas JA Colon water transport in transgenic mice lacking aquaporin-4 water channels. Am J Physiol Gastrointest Liver Physiol 279 G463-G470, 2000.

23. Zhi H and Yuan WT: Expression of aquaporin 3,4, and 8 in colonic mucosa of rat models with slow transit constipation. Zhonghua Wei Chang Wai Ke Za Zhi 14: 459-461, 2011 (In Chinese).

24. Wang XJ, Yuan WT, Song JM and Zhang ZY: Expression and significance of aquaporin 4 in the colonic mucosa of patients with slow transit constipation. Zhonghua Wei Chang Wai Ke Za Zhi 13: 445-447, 2010 (In Chinese).

25. Silberstein C, Kierbel A, Amodeo G, Zotta E, Bigi F, Berkowski D and Ibarra C: Functional characterization and localization of AQP3 in the human colon. Braz J Med Biol Res 32: 1303-1313, 1999.

26. Ikarashi N, Kon R, Iizasa T, Suzuki N, Hiruma R, Suenaga K, Toda T, Ishii M, Hoshino M, Ochiai W and Sugiyama K: Inhibition of aquaporin-3 water channel in the colon induces diarrhea. Biol Pharm Bull 35: 957-962, 2012.

27. Zhang WS, Li F and Bao JQ: Regulatory effect of anthraquinone derivatives from rhubarb on aquaporin 4 expression in colon of rats and in LoVo cell line. Zhongguo Zhong Xi Yi Jie $\mathrm{He} \mathrm{Za}$ Zhi 28: 818-823, 2008 (In Chinese).

28. Matsuzaki T, Tajika Y, Ablimit A, Aoki T, Hagiwara H and Takata K: Aquaporins in the digestive system. Med Electron Microsc 37: 71-80, 2004.

29. Koyama Y, Yamamoto T, Tani T, Nihei K, Kondo D, Funaki H, Yaoita E, Kawasaki K, Sato N, Hatakeyama K and Kihara I: Expression and localization of aquaporins in rat gastrointestinal tract. Am J Physiol 276: C621-C627, 1999.

30. Hoque AT, Yamano S, Liu X, Swaim WD, Goldsmith CM, Delporte $\mathrm{C}$ and Baum BJ: Expression of the aquaporin 8 water channel in a rat salivary epithelial cell. J Cell Physiol 191: 336-341, 2002.

31. Wang JQ, Zhang L, Tao XG, Wei L, Liu B, Huang LL and Chen YG: Tetramethylpyrazine upregulates the aquaporin 8 expression of hepatocellular mitochondria in septic rats. J Surg Res 185: 286-293, 2013. 